H. Magnussen

A. Hellmann

\section{Die Deutsche Gesellschaft für Pneumologie und der Berufsverband der Pneumologen vereinbaren enge Kooperation}

\author{
German Society of Pneumology and Association of German Pneumologists \\ Improve their Cooperation
}

\begin{abstract}
Die Finanzbasis des Gesundheitssystems ist an seine Grenzen gestoßen, Altersentwicklung, Ansprüche der Patienten und Ärzte an das Gesundheitssystem und eine explodierende medizinische Leistungsfähigkeit fordern als Preis einen immer größeren Teil des Einkommens derer, die noch in Lohn und Brot stehen. Die Grenze der Belastbarkeit unserer Volkswirtschaft scheint erreicht. Die Pneumologie spielt zwar eine wichtige Rolle innerhalb der Medizin, steht aber im Wettbewerb mit anderen Ansprüchen an die finanziellen Ressourcen. Jeder spürt, dass der Veränderungsdruck wächst. Die jüngst bekanntgewordenen Vorschläge der Bundesregierung lassen erkennen, dass die Zeit der Rücksichtnahme zu Ende geht.
\end{abstract}

Wie muss sich nun die Pneumologie entwickeln, um im Zuge der Umstrukturierungen der Medizin zu bestehen?

Die klinische Erfahrung, die Bewertung des Sachverständigenrates der Bunderregierung, die Einschätzung der WHO und eine Fülle wissenschaftlich untermauerter epidemiologischer Daten zeigen eindrucksvoll, dass Erkrankungen der Lunge und der Atemwege eine zunehmende medizinische und volkswirtschaftliche Bedeutung haben. Die Pneumologen und ihre Fachgesellschaft könnten sich daher in der Hoffnung wiegen, dass der Zwang des Faktischen zur Zukunftssicherung beitragen wird. Diese logisch anmutende Schlussfolgerung ist trügerisch! Welche Gefahren drohen uns?

Pneumologischen Sachverstand gibt es im ambulanten und stationären Versorgungsbereich. Trotz der vernünftigen Aufgabenteilung, die sich aus den unterschiedlichen Anforderungen entwickelt haben, sind nicht unwesentliche Kräfte der Gesundheitspolitik der Meinung, dass Fachärzte in der ambulanten und stationären Medizin einer Doppel- und damit Überversorgung gleichkommen. Der ambulante Teil der pneumologischen Versorgung wird als überflüssig dargestellt.

Eine derartige Entwicklung ist gefährlich, da bereits jetzt weder die pneumologischen Praxen noch die Fachkliniken die Flut der Patienten zu bewältigen vermögen. Es ist nicht erkennbar, dass die Patienten den Pneumologen aus Verlegenheit aufsuchen, sondern in der Regel aus der selbst erfahrenden Notwendigkeit, ihre Leiden durch mehr Fachkompetenz zu lindern. Die wissenschaftliche (internationale) Literatur belegt, dass die Betreuung pneumologischer Erkrankungen durch den Spezialisierten zu mehr Lebensqualität und geringeren Kosten führt, als die Diagnostik und Therapie durch den weniger erfahrenen, nicht spezialisierten Arzt. Die deutschen Pneumologen sind aufgerufen, diesen Zusammenhang auch für das deutsche Gesundheitswesen zu belegen.

Die Disease Management Programme (DMP) werden ein wichtiger Prüfstein sein, um zu erkennen, ob es der Politik darum geht, die Qualität der Versorgung tatsächlich zu verbessern, oder ob es um vordergründige Kostenreduktion und Deprofessionalisierung geht. Diese Grundfrage stellt sich auch für die weiteren traditionellen Felder der Pneumologie.

Die Pneumologie ist ohne Zweifel ein attraktives Teilgebiet der Inneren Medizin und führt zahlreiche Nachbardisziplinen in die Versuchung, die Pneumologie zu ihren Gunsten zu zerlegen. Die Diskussionen und der Streit um die neue Weiterbildungsordnung ist dafür ein beredtes Zeugnis. Im ersten Entwurf dieser Weiterbildungsordnung wurde uns weder die Kompetenz zur Therapie onkologischer-fachbezogener Erkrankungen noch die Diagnostik und Therapie schlafbezogener Erkrankungen (um nur einige Beispiele zu nennen) zugestanden. Eine intensive Ko- 
operation der DGP und des BdP hat erfreuliche und dringend notwendige Änderungen bewirkt. Wir werden gemeinsam alle Anstrengungen unternehmen, um weitere unverzichtbare Tätigkeiten (fachbezogene Radiologie) zu erhalten und verbleibende Ungereimtheiten zu beseitigen. Der Schulterschluss von DGP und BdP ist eine Lehrstunde für die Effektivität von Pneumologen, die sich für ihr Fach einsetzen und die überflüssigen Gräben zwischen ambulanter und stationärer Medizin überwinden.

Die Krankenhäuser werden sich in der neuen Welt der DRGs zurecht finden müssen. Wir sollten jedoch nicht dem Irrtum unterliegen, dass DMP nur die ambulante und DRG nur die stationäre Versorgungsebene betreffen. Man wird von uns erwarten und verlangen, dass wir Konzepte anbieten, die die Grenzen zwischen ambulanter und stationärer Versorgung überwinden. Dabei ist es wesentlich, dass wir diese Strukturen gemeinsam entwickeln und dass wir sie gemeinsam umsetzen. Nur so wird die Akzeptanz zu gewinnen sein, die für einen Erfolg unabdingbar ist.

Die pneumologische Rehabilitation ist ein effektiver und daher unverzichtbarer Teil des peumologischen Therapieangebotes. Die Rehabilitation kann stationär, teilstationär oder ambulant erfolgen. Die Wahl eines Angebotes sollte sich aus der Situation des
Patienten ergeben und nicht aus der Verfügbarkeit regionaler Rehabilitationseinrichtungen. Nur wenn die Öffentlichkeit erkennt, dass die Verbreiterung eines medizinischen Angebotes keinen standespolitischen Interessen, sondern wissenschaftlichen Erkenntnissen folgt, können wir auf Akzeptanz hoffen.

Als kleines Spezialgebiet im Gesamtkontext der Medizin lebt die Pneumologie mehr als andere von den Fortschritten der Wissenschaft auf der einen Seite und der Relevanz für die Patientenversorgung auf der anderen Seite. Entscheidend aber dabei ist, wie gut beides vernetzt ist und wie schnell die Umsetzung an die Basis erfolgt. Dafür werden wir in Zukunft gemeinsam arbeiten.

Wir Pneumologen sind in verschiedenen Gesellschaften organisiert. Die unterschiedlichen Schwerpunkte der mehr wissenschaftlich orientierten DGP und des mehr berufspolitisch orientierten BdP dürfen jedoch nicht darüber hinwegtäuschen, dass wir alle ein gemeinsames Ziel verfolgen: den Erhalt und die Fortentwicklung der Pneumologie.

DGP und BdP werden durch ihre Vertreter in den jeweiligen Vorständen eine strategische Einheit bilden und gemeinsame Projekte entwickeln, um unsere berechtigten Vorstellungen durchzusetzen. 